\title{
Los pro y contra de la legalización de la marihuana
}

\author{
FERNANDO MÖNCKEBERG B. ${ }^{1}$
}

1. Universidad Diego Portales.

\begin{abstract}
\section{Pros and cons of legalizing marijuana}

There are already several countries that have accepted marijuana as a soft drug, separating it from more dangerous ones. Various therapeutic properties have even been attributed to its use. Others, however, think that its use should be prohibited due to the mental interference and behavioral changes produced either by its occasional use as well as the permanent mental damage linked to chronic marijuana use. In order to clarify this divergence of opinions, the scientific literature is reviewed. It is concluded that there is a serious risk, especially for teenagers, associated to chronic marijuana use due to the presence of more frequent psychotic and schizophrenic episodes, which can be permanent, while consumption during pregnancy results in brain damage to the fetus, similar to fetal alcohol syndrome. Scientific research also indicated that smoking marijuana produces an even more severe bronchial damage than tobacco, with risk of lung cancer. In conclusion, the notion that marijuana is a risk-free soft drug is a serious mistake, based on the available conclusive scientific research that shows the opposite.
\end{abstract}

(Key words: Marijuana, drug, drug use, soft drug, tetrahydrocannabinol).

Rev Chil Pediatr 2014; 85 (2): 229-237

\section{RESUMEN}

Ya son varios los países que han aceptado la marihuana como una droga "blanda", diferente a otras más peligrosas. Incluso se le ha atribuido diversas propiedades terapéuticas. Otros sin embargo piensan que su uso debería prohibirse, dadas las interferencias mentales y las alteraciones del comportamiento que produce, ya sea por su consumo esporádico, como también por los daños permanentes a que lleva su consumo crónico. Con el objeto de aclarar estas divergentes apreciaciones, se revisa la literatura científica existente. De ello se deduce el grave riesgo de su consumo crónico, especialmente para el adolescente por el significativo incremento de cuadros psicóticos y esquizofrénicos, los cuales pueden llegar a ser permanentes, mientras que su consumo durante el embarazo daña el desarrollo cerebral del feto en forma similar a lo que hace el alcohol en el síndrome fetal alcohólico. Investigaciones científicas también señalan que al fumar sus hojas, se va produciendo un daño bronquial, aún más grave que con el tabaco, incluyendo el cáncer pulmonar. En conclusión, el concepto de que la marihuana es una droga "blanda", libre de riesgos para la salud, constituye un grave error, dado la concluyente investigación científica disponible que señala lo contrario.

(Palabras clave: Marihuana, droga, consumo de drogas, droga blanda, tetrahidrocanabinol).

Rev Chil Pediatr 2014; 85 (2): 229-237

Recibido el 26 de marzo de 2014, aceptado para publicación el 12 de abril de 2014.

Este trabajo cumple con los requisitos sobre consentimiento /asentimiento informado, comité de ética, financiamiento, estudios animales y sobre la ausencia de conflictos de intereses según corresponda.

Correspondencia a:

Fernando Mönckeberg B.

E-mail: fernandomonckeberg@conin.cl 


\section{Introducción}

Según Naciones Unidas, la marihuana es la substancia ilícita más utilizada en el mundo. En su informe oficial afirma que más del $4 \%$ de la población adulta mundial (162 millones de personas) la consume más de alguna vez durante el año, mientras que el 0,6\% (22 millones) la consume a diario. Por otra parte estima que en la actualidad unos 20 millones de personas en el mundo son adictas a ella ${ }^{1}$.

En la última encuesta nacional realizada en los Estados Unidos, se señala que el $48 \%$ de los americanos alguna vez la ha aprobado y que el 6,5\% de los estudiantes de "high school", admite consumirla regularmente. Por ello no asombra que en un esfuerzo por controlar su consumo, en noviembre de 2012, dos estados (Washington y Colorado) la hayan legalizado, permitiendo su consumo en cantidades limitadas y sólo en los mayores de 21 años ${ }^{2}$. Por su parte, los activistas esperan que pronto otros estados también la aprueben.

Ya anteriormente, y con las mismas razones, Holanda (1976) había autorizado la venta a mayores de 18 años, pero restringida sólo a locales específicos, llamados "Coffee Shop", donde se podía vender hasta en cantidades de $5 \mathrm{~g}$. Estos rápidamente se multiplicaron y en la actualidad ya sobrepasan los 900, que no tienen mucho de "Coffee", pero sí de expendio legal de marihuana. Lo paradójico es que a estos mismos locales no les está permitido comprar marihuana, ya que su cultivo no está legalizado. "Es que modificar esta última restricción tendría consecuencias negativas, dado que entraría a incidir el factor "mercado" que presionaría el consumo, como sucede con el tabaco. En todo caso, la autorización de los Coffee Shop ha traído otros problemas en los que no se había pensado. Los menores de 18 años, incentivados por el consumo de sus mayores, van ahora buscarla a lugares más apartados y menos regulados. Según el Instituto Timbos, un centro de estudio de la adición en Utrecht, los menores allí han incrementado su consumo abasteciéndose por medio de distribuidores mafiosos ${ }^{3}$. Con ello, aparte del daño que se puede provocar en sus cerebros, está también el que se produce en sus pulmones, dado que la marihuana la venden por peso, por lo que frecuentemente le agregan partículas de vidrio, cenizas, tizas o arena. Al respirar crónicamente estos ingredientes se dañan sus pulmones, produciendo escaras en su interior. Otro ingrediente detectado en la adulteración ha sido el agregado de partículas de plomo, que al aumentar significativamente su peso específico, incrementa también las utilidades de los inescrupulosos traficantes. La intoxicación por plomo agregado a la marihuana se comprobó en Leipzig, Alemania, donde en el año 2008 treinta personas presentaron simultáneamente síntomas de intoxicación aguda, que los obligó acudir a los hospitales de la ciudad. Allí sus síntomas hicieron sospechar una intoxicación por plomo. Sus edades oscilaban entre 16 y 33 años, y todos tenían de común el haber inhalado marihuana adquirida en la calle. Analizada algunas muestras se comprobó que contenía un $10 \%$ de partículas de plomo ${ }^{4}$. Posteriormente, tratando de averiguar más, se tomaron muestras de sangre al azar a 145 personas conocidas consumidoras de marihuana, comprobándose que 95 de ellas tenían niveles tan altos de plomo, como para requerir tratamiento. Simultáneamente se demostró que el plomo se absorbía muy efectivamente por el tracto respiratorio. Como conclusión los autores recomiendan que frente a hallazgos de niveles sanguíneos elevados de plomo en la sangre, se deba considerar a la marihuana adulterada como una potencial fuente de ese metal ${ }^{4}$.

En Inglaterra, en el año 2004 una comisión gubernamental decidió pasar la marihuana de la clase $\mathrm{B}$ a la clase $\mathrm{C}$, dentro de un índice que señala la peligrosidad de las drogas, estimando que ella no debía pertenecer a la misma categoría que la cocaína y las anfetaminas, que eran mucho más peligrosas. Fue a raíz de esta decisión que el Ministerio del Interior Británico decidió su despenalización, aun cuando mantenía las penas por 14 años de prisión para aquellos que poseyeran la droga con intención de traficar con ella. Esta reforma fue duramente criticada tanto desde el punto de vista político, como de salud. Fue así como Keit Halliwell, asesor político del gobierno, renunció a su cargo ya que consideró que la liberación iba a ser tomada por la juventud como un mensa- 
je que las drogas no son malas. Mientras tanto que por otro lado, Louise Arseneault, psiquiatra investigadora del Instituto de Psiquiatría del King College en Londres, afirmaba que ya eran consistentes las investigaciones que demostraban que el uso de marihuana, especialmente en adolescentes, causaba problemas mentales a largo plazo, con un significativo mayor riesgo de producir esquizofrenia ${ }^{5}$. Ante la reacción pública y en respuesta a ella, Charikes Clark, ministro del interior prometió revisar la clasificación de la droga.

En Uruguay, en diciembre 25 de 2013, se publicó la ley que permitía comprar $40 \mathrm{~g}$ de marihuana por mes a cada habitante mayor de 18 años. Ella permitía además la posibilidad de cultivo de marihuana hasta 6 plantas por familia, con una cosecha no mayor de $4.809 \mathrm{~g}$. Por esta razón, y porque además se había legalizado en el mismo año el matrimonio entre homosexuales, la revista "The Economist", eligió a Uruguay como el país del año 2013 (The Economist, 19 dic. 2013).

En otros países, en que no se permite el consumo, existe una variada legislación, según sea el tráfico, el cultivo o la posesión de ella. En Chile el consumo personal es legal, pero es ilegal el cultivo, la producción, la compra y su comercialización. Según la encuesta del consumo de drogas de 2012, el 17,5\% de los jóvenes entre 19 y 25 años reconoció haber consumido esta substancia. Ello puede cambiar a futuro, dado que con fecha de Mayo del 2013, se envió al Congreso un proyecto de ley que despenaliza la marihuana. Este se encuentra actualmente en discusión de la Comisión de Salud del Senado.

\section{Mecanismo de acción de la marihuana}

Lo que no se puede negar es que la marihuana lleva a profundos cambios en el comportamiento y la conciencia del consumidor. El principal componente psicoactivo de la marihuana, el tetrahidrocanabinol (THC) fue descubierto por Raphael Mechoulam de la Universidad Hebrea de Jerusalén ${ }^{6}$. El mismo, algún tiempo más tarde descubrió que el cerebro producía una substancia química que actuaba en forma similar al THC de la marihuana. Se trataba de un pequeño ácido graso que denominó anandamida. Poco tiempo después se descubrió otro lípido natural activo, el 2-araquidonil glicerol $(2 \mathrm{AG})^{7}$. Tanto la anandamida como el $2 \mathrm{AG}$, producidos por las células del organismo, pasaron a llamarse endocanavinoides, a diferencia del canabinoide propio de la marihuana $^{8}$.

Posteriormente, se pudo demostrar que los endocanavinoides, para ejercer su acción en el interior de las neuronas, debían ser previamente reconocidos por una proteína receptora localizada en la membrana neuronal. A esta se le conoce como "proteína receptora de canabinoides", dado que reconoce tanto a los endocabinoides (anandamida y 2AG) como el THC producido por la marihuana.

Llama la atención que siendo tan diferentes las estructuras químicas de los endocanabinoides (anandamida y 2AG), con respecto al THC de la marihuana, calcen con los mismos receptores (figura 1). El hecho es que el THC al ser reconocido por estos receptores específicos, ubicados en la pared de las neuronas, parece desplazar y competir en sus acciones. Cabe señalar que ellos son los receptores neuronales

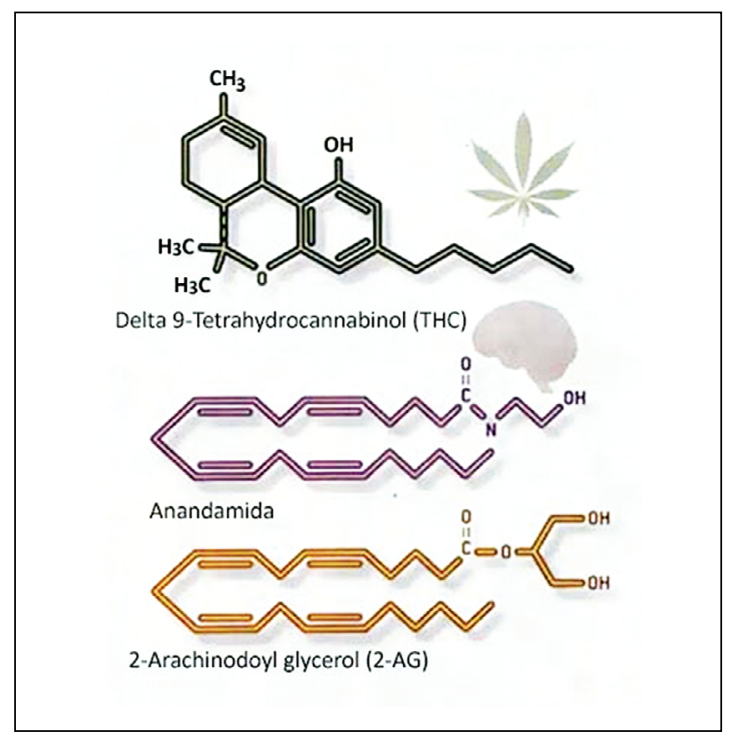

Figura 1. Estructura de las exo y endocanabinoides. A pesar de la diferencia estructural del THC, producido por la marihuana y la anandamida y $2 \mathrm{AG}$, producidas por el cerebro, activan los mismos receptores. 


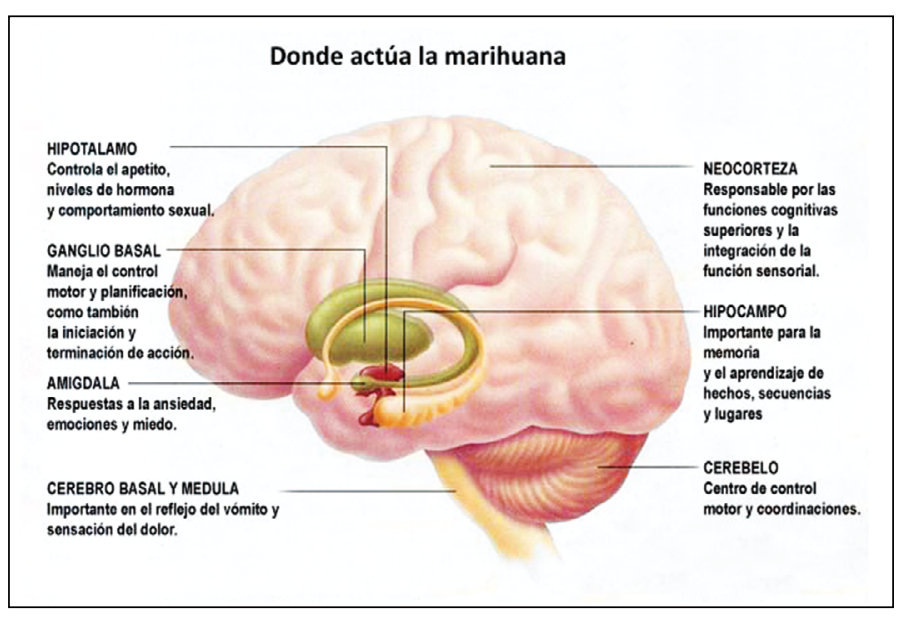

Figura 2. La droga "Cannabis sativa" se une a los receptores celulares que tienen los endocanabinoides en las neuronas, ubicadas en diferentes partes del cerebro. más abundantes, encontrándose ampliamente distribuidos en el cerebro. Se encuentran en las células del hipocampo, hipotálamo, cerebelo, ganglio basal, amígdala, tronco cerebral y en la médula ósea (figura 2). Ello explica la diversidad de síntomas clínicos que produce la marihuana, dado que en cada ubicación parecen competir con los mismos receptores de los endocanabinoides, interfiriendo en sus propias acciones metabólicas.

$\mathrm{Su}$ acción intracelular se traduce en un incremento de la producción de neurotransmisores (las moléculas que las células neuronales usan para comunicarse entre sí). Con ello el usuario se siente eufórico, o "high", ya que el THC actúa especialmente en el sistema cerebral de gratificación, del mismo que lo hacen casi todas las otras drogas. Para ello el THC estimula a las células cerebrales para que liberen una sustancia química llamada dopamina. El usuario se siente alegre, relajado, y curiosamente comienza a encontrar muy agradable el sabor de los alimentos. La euforia pasa después de un tiempo y comienza a sentir sueño o depresión. En ocasiones su ingestión produce ansiedad, temor, desconfianza, y no raras veces desencadena una crisis de pánico ${ }^{9}$.

\section{Efecto inmediato de la marihuana}

Consumida esporádicamente y en pequeñas dosis, altera la función de la memoria, distorsiona la percepción y disminuye las habilidades motoras. Es así como la marihuana afecta diversas capacidades mentales, especialmente la memoria y la atención. En numerosos trabajos se ha demostrado que las personas bajo la influencia de la marihuana rinden deficientemente en las evaluaciones de la llamada "memoria de trabajo", que es la habilidad relacionada con las estructuras y procesos utilizados por la mente para el almacenamiento de información y la simultánea manipulación de ella ${ }^{10}$.

Durante los test para revalidar permisos de conducir se ha observado que los que están bajo el efecto de la marihuana, tienen grandes dificultades para recordar una lista de palabras tomadas al azar. Se les dificulta la concentración y se les debilita la coordinación motora, especialmente cuando se trata de sortear obstáculos. Incrementa los tiempos de reacción, siendo más lentos para aplicar los frenos y altera la percepción de las distancias. Estos déficits cognitivos no son de trascendencia si se está sentado en la casa, pero sí lo son cuando se maneja un automóvil o pilotea un avión. Allí son causa de accidentes fatales. Su efecto deletéreo dura cuatro a seis horas después de haber fumado un solo cigarrillo.

En un estudio realizado en el año 2009, se demostró que el $\mathrm{TCH}$, con dosis mayores a cinco nanogramos por milímetro de sangre, ya incrementa significativamente los riesgos de accidentes de tránsito, lo que es equivalente 
a una alcoholemia de 0,08\%. En la actualidad ello es especialmente preocupante, ya que de acuerdo a un informe de la American Society of Addition Medicine, en la actualidad se ha logrado producir plantas de marihuana con una mayor concentración de TCH y ellas ya están en el mercado. En el año 1960, en USA el promedio de cigarrillos de marihuana contenían 10 milígramos de THC. Hoy en día estos mismos contienen $150 \mathrm{mg}^{2}$.

Para complicar más las cosas, la detección policial de la marihuana, a diferencia del alcohol, no se puede realizar por un simple test de inhalación. Para comprobar su consumo la policía tendría que tomar muestra de sangre y enviarla a un laboratorio, lo que hace el procedimiento impracticable. El uso de la marihuana se puede oler al interior del automóvil, pero ello es una detección subjetiva.

Es frecuente que la ingestión de una alta dosis de marihuana desencadene una psicosis aguda, con alucinaciones, delirio y pérdida del sentido de identidad. Ello ocurre cuando se ingiere la droga directamente en lugar de fumarla. Estas crisis son distintas a los trastornos de mayor duración, semejantes a la esquizofrenia, que se asocia al uso continuo de la droga ${ }^{11}$.

\section{Efecto a largo plazo de la marihuana}

Se ha observado que el consumo de la marihuana en forma crónica llega a producir alteraciones cerebrales permanentes, las que son más graves si el consumo se ha iniciado tempranamente, durante la adolescencia, cuando aún no se ha completado el desarrollo cerebral. Se han descrito anomalías cerebrales estructurales, como disminución del volumen en la zona del hipocampo y la amígdala ${ }^{12}$, así también como reducción del volumen cerebral ${ }^{13}$. Mientras tanto, se ha descrito que la administración de la marihuana en ratas, modifica la producción de neuronas en el hipotálamo (zona en que radica la memoria), tanto en el embrión, como también en la rata adulta ${ }^{14}$.

Estos hallazgos son importantes, dado que de acuerdo al National Institute of Drug Abuse del NIH (USA), el uso mantenido de marihuana es aditivo ${ }^{2}$. En adultos que consumen mari- huana a largo plazo, un 10\% llega a ser adicto. El porcentaje es significativamente mayor en los que comienzan a utilizarla tempranamente, ya durante la adolescencia (25\%). Más aún en los que la consumen directamente (58\%de los casos) ${ }^{15}$. Ya anteriormente Steven Goldberg y sus colaboradores del NIH habían demostrado en monos, que al igual que la cocaína y la morfina, la marihuana era adictiva. Lo comprobaron mediante un dispositivo en que cada vez que el mono tocaba un botón, automáticamente se inyectaba THC. Rápidamente ellos aprendieron y se inyectaban la substancia hasta treinta veces en cada sesión. Más tarde, al darle una droga que bloqueaba el receptor para marihuana en el cerebro (rimonabant), los monos dejaban de presionar el botón ${ }^{16}$.

Las personas que intentan dejarla, presentan irritabilidad, dificultad para dormir, deseo vehemente por la droga y ansiedad. Simultáneamente desarrollan agresividad que llega a su punto máximo a la semana después de haber dejado la droga ${ }^{15}$.

El consumo crónico durante la adolescencia produce un daño cerebral muy semejante a lo que va ocurriendo durante la vejez, en que se van perdiendo neuronas en el hipocampo, con lo que disminuye la capacidad de aprender información nueva. Al comienzo, en el adolescente, los síntomas se manifiestan sólo en cambios de humor, pero después de un tiempo se va llegando a una apatía progresiva, falta de iniciativa, carencia de demostraciones afectivas, disminución de la capacidad de concentración y con ello se va produciendo una progresiva disminución del rendimiento escolar. Ello es la expresión del daño progresivo de la estructura cerebral del hipocampo.

Una pregunta recurrente es si los fumadores crónicos, que la consumen desde la adolescencia, pueden o no recuperar algunas de sus capacidades cognitivas dañadas si dejan de consumirla. La respuesta no es definitiva, dado que lo observado proviene generalmente de experiencias animales. Pero basados en ellas, se concluye que los daños pueden acumularse y con el tiempo llegar a deteriorar las habilidades cognitivas en forma definitiva. Así por ejemplo, en ratas expuestas al THC in útero, o poco después del nacimiento, o aún durante 
la adolescencia, se detectan problemas importantes en tareas específicas de aprendizaje y en su memoria ${ }^{12}$. En humanos los hallazgos son más difíciles de comprobar, dado los prolongados tiempos de observación necesarios y la necesidad de grupos lo suficientemente numerosos como para sacar conclusiones valederas. Sin embargo, en un reciente estudio realizado por la psicóloga Madeleine Meier y sus colegas de la Universidad de Duke, pudieron seguir los daños posteriores en un control prospectivo en 1.037 jóvenes neozelandeses, consumidores de marihuana. Las investigaciones se fueron realizando a través de controles sucesivos en la medida que iban cumpliendo 18, 21,26, 32 y 38 años de edad ${ }^{17}$. Los investigadores encontraron que aquellos que comenzaron a fumar marihuana precozmente y con mayor frecuencia, al cumplir 38 años de edad, habían experimentado una disminución de ocho puntos, como promedio, en su cociente intelectual. Más tarde, al cesar el uso de la marihuana no se restablecieron completamente las funciones neuropsicológicas. Las autoras concluyen que la marihuana tiene un efecto neurotóxico en el cerebro del adolescente y por ello llaman la atención en la necesidad de prevención, ya que el daño sufrido tempranamente en la adolescencia, dejaría secuelas definitivas ${ }^{15}$. Otros estudios no parecen concluyentes, ya sea por la presencia de diversos factores intervinientes o por la agregación de deterioros propios de los procesos mentales, como el envejecimiento ${ }^{10}$.

Ya hay suficiente evidencia que el consumo de marihuana en gran cantidad llega a producir síntomas propios de una psicosis, como desorientación, despersonalización, paranoias y alucinaciones, mientras que su uso continuo llega con frecuencia a producir esquizofrenia. Esta asociación entre marihuana y esquizofrenia fue descrita por primera vez en Suecia, en la década de 1980, cuando se examinaron las historias clínicas de 50.087 jóvenes que habían hecho el servicio militar entre 1969-1970. Al revisar sus fichas, los autores observaron que aquellos que declararon haber fumado marihuana (en más de 50 ocasiones), presentaron más tarde los síntomas de esquizofrenia con una frecuencia seis veces mayor que los que no habían fumado marihuana ${ }^{18}$. Ello ha sido confirmado posteriormente por Mary Cannon y colaboradores del Royal College of Surgeons en Irlanda, que realizó un estudio de seguimiento de 759 personas en Dunedin, Nueva Zelanda, durante los años 1972 y 1973. Los autores concluyen que los que han fumado marihuana antes de los 15 años son más susceptibles de desarrollar esquizofrenia en edades posteriores, en relación a los que no han fumado ${ }^{19}$. Resultados similares se han descrito también en Grecia, donde se concluye que las personas susceptibles a la esquizofrenia, si consumen marihuana, tienen un 50\% de mayor riesgo de llegar más tarde a presentar síntomas de esquizofrenia. Mientras más cigarrillos de marihuana fuman, y a más temprana edad lo hacen, más incrementan el riesgo ${ }^{20}$. Otras publicaciones más recientes, confirman que existe una minoría de personas que genéticamente tienen una predisposición a la psicosis y esquizofrenia y que a ellas es muy conveniente aconsejarles que se mantengan lejos de la marihuana. Desgraciadamente a ellas no hay como detectarlas. En todo caso son numerosas las publicaciones de metanalisis que asocian el consumo mantenido de marihuana con la esquizofrenia o con diferentes tipos de psico$\operatorname{sis}^{21-25}$. El último informe del National Institute on Drug Abuse de USA, enfatiza que cualquiera sean los daños cerebrales detectados, el consumo crónico de marihuana, siempre está asociado a problemas de la vida diaria. Ellos tienen menos satisfacciones, peores condiciones de vida y de salud, dificultades sociales, mayores índices de deserción, menores niveles educacionales y profesionales, en relación a sus pares ${ }^{26}$.

Pero la marihuana no sólo produce daños en el cerebro, sino también en otros órganos, y muy especialmente, en los pulmones. Diversas publicaciones señalan que del riesgo de desarrollar un cáncer pulmonar en fumadores de marihuana, es incluso más elevado que en los fumadores de tabaco. En Nueva Zelandia, donde existen los más altos porcentajes de fumadores de marihuana, y también tienen los más altos índices de cáncer del pulmón, Steven Aldington y sus colaboradores del Medical Research Institute, han tenido la oportunidad 
de estudiar este riesgo, concluyendo que los fumadores diarios de marihuana incrementan en un $8 \%$ al año el riesgo de sufrir cáncer pulmonar, llegando a superar el riesgo de los que se fuman un paquete de cigarrillos al día durante un año ${ }^{27}$. A similares conclusiones habían llegado también otros investigadores, y la British Lung Foundation, agregando que cuando se fuma marihuana y tabaco, el riesgo de cáncer se incrementa aún más ${ }^{28-35}$, por lo que se concluye que debería alertarse a las autoridades para que implementen con la marihuana las mismas o mayores regulaciones que se han estado implementando para disminuir el tabaquismo ${ }^{36}$. Si bien es cierto que el humo de la marihuana es similar al del tabaco, el primero contiene dos veces mayor concentración de hidrocarbones poliaromáticos carcinogénicos ${ }^{28}$, con el agravante que los cigarrillos de marihuana no tienen filtros y los usuarios tienden a inhalar más profundamente, manteniendo el humo en los pulmones por más tiempo (de promedio, tres veces más). Por otra parte, el alquitrán de los cigarrillos de marihuana, contienen un 50\% más de benzatracenos y benzapirenos, que son carcinogenénicos ${ }^{33}$. Diversos estudios han demostrado también en el tracto respiratorio de fumadores de marihuana, la existencia de anormalidades histológicas y moleculares precancerosas $^{36,37}$, mientras que se ha demostrado in vitro y en diferentes modelos animales los efectos carcinogenéticos del humo de marihuana $^{27,39}$.

Especial mención merecen los antecedentes del efecto de la marihuana durante el embarazo, ya que diversos antecedentes señalan que afecta al desarrollo fetal. En madres consumidoras de marihuana se ha descrito en sus hijos un bajo peso al nacer y una menor talla, con daños semejantes a lo que produce la ingesta de alcohol (síndrome fetal alcohólico) ${ }^{40}$. Parece ser que la marihuana durante el embarazo altera el desarrollo del sistema vascular del feto en la misma forma que lo hace el alcohol ${ }^{41}$ Se ha descrito también que la marihuana afecta el desarrollo neuronal fetal, lo que podría explicar alteraciones posteriores en el comportamiento $^{42}$. La exposición a la marihuana durante el embarazo se asociaría con posteriores problemas del comportamiento detectables ya a los 18 meses de edad, especialmente en niñitas $^{43}$.

\section{Uso médico o terapéutico de la marihuana}

La mayor parte de las drogas usadas en la práctica médica han tenido su origen en las plantas y/o microorganismos. Ha sido la medicina tradicional, que detectando sus cualidades curativas ha ido traspasando la información de generación en generación. Por lo general, se trata de compuestos químicos de estructuras demasiado complejas, que con muy baja probabilidad podrían haber sido descubiertas desde el laboratorio. Por el contrario, ha sido este el que ha aprovechado la química de la naturaleza, que habiendo evolucionando a lo largo de millones de años, ha ido creando complejas estructuras químicas en un esfuerzo constante de llegar a hacer posible y sustentable la vida biótica. Allí el hombre pudo ir conociendo a través de su acción los más diversos fármacos que las plantas contenían y que hoy utiliza la medicina: anestésicos, narcóticos, vasodilatadores, dilatadores, estimuladores respiratorios, relajadores musculares, agentes colinérgicos, antibióticos y muchos otros. El procedimiento está lejos de haberse agotado. Se estima que existen en la naturaleza aproximadamente unas 250 mil especies de plantas con flores y hasta ahora sólo se han examinado no más de 5 mil. Hoy como ayer, ellas continúan asombrando con sus complejas estructuras químicas y en ellas el hombre busca posibles acciones terapéuticas.

La marihuana (Cannabis sativa) no es una excepción. Sus efectos psicotrópicos se conocen desde siglos antes de Cristo y en sus células se han identificado 483 componentes, entre los cuales 66 son catalogados como cannabinoides ${ }^{44}$. En los últimos años diversas investigaciones han demostrado la influencia de los endocanabinoides y sus receptores celulares específicos en una variedad de procesos fisiológicos y patológicos. Es por ello que la industria farmacológica se ha demostrado tan interesada en desarrollar fármacos capaces de potenciar o anular sus acciones. Ya se conoce una enorme variedad de acciones y sus influencias en diferentes procesos fisiológicos, como 
el aprendizaje, la memoria, la regulación del apetito, y en el metabolismo. A ello se agregan los efectos descritos en las emociones, como el miedo y la ansiedad, la inflamación, el crecimiento óseo e incluso el desarrollo de ciertos cánceres, entre otras acciones.

Las diversas acciones psicobiológicas producidas por los canabinoides ha llevado a buscar drogas que incrementen su acción, como también otras que las inhiban, según el caso. Es así como por se descubrió el Rimonabant, un inhibidor de endocanabinoides ${ }^{45}$, cuyo uso se ha prohibido en algunos países, después de comprobar la aparición indeseada de trastornos psiquiátricos graves, depresiones e incluso suicidios en quienes lo ingerían.

Sin duda que productos derivados de la marihuana pueden llegar a ser fármacos muy útiles en la medicina moderna. Para facilitar su desarrollo no se requiere liberalizar su consumo. La industria farmacéutica puede disponer de permisos para su cultivo controlado y de ella aislar y purificar el ingrediente activo para utilizarlo como un producto farmacológico, ya sea en grageas, gotas, inhalación u otras formas que permitan su administración dosificada, como sucede con los muchos otros fármacos derivados de plantas. Es por ello que en casi todo los países se ha autorizado el uso médico de la marihuana, pero no se ha permitido el uso recreacional de ella. Tal es el caso por ejemplo de USA, donde si bien es cierto que recientemente se ha permitido el uso recreacional de la marihuana en dos estados ${ }^{10}$, en los otros diez y seis sólo se ha autorizado su uso medicinal. En la legislación chilena se sanciona la producción, fabricación, elaboración, distribución, posesión o tenencia de marihuana, pero el consumo médico está autorizado. Por otra parte, la ley $\mathrm{N}^{\circ} 20.000$ entrega al Presidente de la Republica la facultad de determinar por vía reglamentaria las substancias capaces de producir dependencia y daños a la salud, cuyo control se somete a severas sanciones que allí se establecen.

En resumen, creer que la marihuana es una droga "blanda", libre de riesgos para la salud, cuyo uso puede permitirse, constituye un grave error, dado la concluyente investigación científica disponible que señala lo contrario.

\section{Referencias}

1.- United Nations on Drug and Crime: Cannabis: Why we should care. United Nations 14.- World Grug Report 2006 vol 1 ISBM 9-2114-3.

2.- National Institute of Drug Abuse: Research Report Series. Marijuana Abuse. Publication US NIH N 10-3859 Julio 2012.

3.- Monshouwery K, Van Dorseelaer S, Verdurmen J, Boqt $T$, Graaf $R$, Valleberrgh $C$ : Cannabis use and mental health in secondary school children. Br J Psychiatry 2006; 188: 148-53.

4.- Busse F, Wrigassen M, Kluge E: Lead poisoning due to dultered mariuana. N Eng J Med 2008; 358: 1641-2.

5.- Arseneault L, Cannon M, Witton J, Murray R: Causal Association between cannabis and psichosis: examination of the evidence. Br J Psychiatry 2004; 84: 110-7.

6.- Mechoulam R, Ben-Shabat L, Legumsky N, Kaminsky $N$, Shatz A, Gopher A: Identification of an endogenous 2-monogliceride, present in cannie gut that bind to cannabinoid receptors. Biochem Pharmacol 1965; 50: 83-90.

7.- Mechoulam R, Shani A, Edery H, Grunfeld Y: The chemical basis of hashish. Science 1970; 169: 611-2.

8.- Devane $W$, Hanus A, Breuer R: Isolation and structure of a brain constituend, that bind to the cannbinoid receptor. Science 1992; 258: 1949-54.

9.- Wilson R, Nicoll A: Endocannabinoid signaling in the brain. Science 2002; 296: 678-82.

10.- Khamsi R: Going to pot. Sci Am 2013; Junio: 34-6.

11.- Fabrant B, Menetrey A, Augsburger L, et al: Cannabis acute psychosis: Psicosis following the administration of oral cannabis. BMC Psychiatry 2005; 50: 17-8.

12.- Yüel M, Salowij N, Respondec C, Whittle S, Fornito A, Pantelis $N$ : Regional brain abnormalities associated with long term heavy cannabis. Arch Gen Psychiatry 2008; 65: 694-701.

13.- Joakes M, Acrome R, Oakes R, et al: Psychological and social sequelae of cannabis and other illicit drug used by joung people: A sistematic review of longitudinal, general population studies. Lancet 2004; 363: 1571-8.

14.- Jiang W, Zhang Y, Xiau L, Van Cleemput J, Shao-Ping $J$, Zhang $X$ : Cannabinoids promote embrionic and adult hippocampus neurogenesis and produce anxiolitic and antidepressant like effect. J Clin Invest 2005; 115: 310416.

15.- National Institute of Health on Drug Abuse: Research Report Series. Marijuana early onset, adition: Cannabis use is linked to IQ decrease: Drug Facts Publication US NIH Agosto 2013. 
16.- Justinova Z, Tauda G, Radhi G, Goldberg S: Self administration of tetrahydrocannabinol (THC) in naive squirrel monckey. Psychopharmacology 2003; 169: 135-40.

17.- Meier M, Caspi A, Ambler A, Harrington H, Houts R, Keef $R$ : Persistant cannabis users show neuropsychological decline from childwood to midlife. Proc Nat Acad of Sc 27 Agosto 2012.

18.- Andréson S, Engström A, Allebeck P, Rydberg U: Cannabis and schizofrenia: a longitudinal study of swedish concrispts. Lancet 1987; 330: 1483-6.

19.- Arseneault L, Cannon M, Poulton R, Murray R, Caspi A, Moffit T: Cannabis use in adolescence and risk for adult psychosis. Brith Med J 2002; 325 (7374): 1212-3.

20.- Lawton G: Cannabis: Two much, to Young? New Scientist 2005; Marzo 26: Magazine issue 2492: 32-4.

21.- Arendt M, Rosemberg R, Foldager L, Perto G, Mun-Jorgensen $P$ : Cannabis-induced psychosis and subsequent shizophrenia-spectrum disorders: Folow-up study of 535 incident cases. Brith J Psychiatry 2005; 187: 510-5.

22.- Mattews L, Swapnil S, Compton M, Slate T, Nielssen O: Cannabis use and early onset of psychosis: A systematic meta-analysis. Arch Gen Psychiatry 2011; 68: 555-61.

23.- Ganzales-Pinto A, Vega P, Ibáñez B, Mosquera F, Barbeito $S$, Gutiérrez $M$ : Impact of cannabis and others drugs on age at onset psychosis. J Clinical Psychiatry 2008; 69: 1210-6.

24.- Le Bec P, Fatséas M, Denis C, Lavie E: Auriacombe M. Cannabis and Psychosis: Search of a causal link through a critical and systematic review. L'Encephale 2009; 35: 377-85.

25.- D'Sauza C: Cannabinoid model psychosis, dopaminecannabinoid interacction and implication for shyzophrenia. En: Marijuana and Madness. Cambridge University Press 2004.

26.- National Institute on Drug Abuse: National Institute of Healh US Researh Report Series. Marijuana Abuse Number: 10-3854, July 2012.

27.- Aldington S, Harwood M, Cox B: Cannabis use and risk of lung cáncer: a case control study. Eur Resoir J 2008; 31: $280-8$.

28.- Callaghan R, Allebeck P, Sidouchuk A: Marihuana use and risk of lung cáncer: a 40 years cohort study. Cancer Causes \& Control 2013; 24: 1811-20.

29.- Ricket W, Robinson J, Royers B: A compartision of tar, carbón monoxide and $\mathrm{pH}$ levels in smock from marijuana and tabaco cigarrete. Cann J Pub Health 1982; 73: 381-91.

30.- Tashkin D, Glieduer F, Rogers B: Tar, CO, and delivery from de 1 st and 2nd halves of marijuana cigarrette. Pharmaco Biochem Behav 1991; 318: 347-51.
31.- Wu T, Taskini D, Rose J: Pulmonary hazard of smoking marijuana as compare with tabaco. N Engl J Med 1988; 381: 347-51.

32.- Mehra R, Moore B, Crothers K, Tetrault J, Feikkini D: The associattion between marijuana smoking and lung cáncer. A systematic review. Arch Inter Med 2006; 166: 1359-63.

33.- Voirin N, Berthiller V, Benhä̈m-Luzon V, Straif B, Ayeb $F$, Sasco A: Risk of lung cáncer and past use of cannabis in Tunesia. J Thorac Oncol 2006; 1: 577-9.

34.- Melaqmeds $R$ : Cannabis and tabacco smoke are not equaly carcinogenic. Harm Reduc J 2005; 18: 2-21.

35.- Taskin D: Smocked narijuana as cause of lung injury Monaldi. Arch Chest 2005; 63: 93-100.

36.- Bramilla C, Colonna M: Cannabis: The next villain on lung cancer battleld. Euro Resp J 2008; 31: 227-28.

37.- Fligiel S, Roth M, Kleeriuf E, Barsky S, Simmon M, Tashkim D: Tracheobronquial histopathology in habitual smockers cocaine, marijuana and/or tabacco. Chest 1997; 112: 319-26.

38.- Gong H, Fligiel S, Taskin D, Tashkin D, Barbers: Tracheo bronchial changes in habitual heavy smockers of marijuana with and without tavacco. An Rev Perpir 1987; 136: 142-9.

39.- Zhu L, Sharma S, Stolina M, Gadner B, Roth M: Delta9-tetrahydrocannabinol inhibits antitumor inmunity by CB2 receptor-mediated, cytokine dependent patway. J Inmunol 2000; 165: 373-80.

40.- Zukerman B, Frenk D, et al: Effect of maternal marijuana and cocaine on fetal growth. N Engl J Med 1989; 32: 762-8.

41.- Marrum N, Tiemer H, et al: A prospectiv study on intrauterine cannabis exposure and fetal growth. Early Human Develop 2010; 86: 231-6.

42.- Gómez M, Hernández-Ruiz J: Prenatal cannabinoid and gene expression for neuronal adhesión molecule L1 in fetal brain. Develop Brain Research 2003; 147: 201-7.

43.- El Marroun H, Hudziak J, Tiemer H, et al: Intrauterine cannabis exposure leads more aggressive behabior and attention problems in 18 month-old-girls. Drug and alcohol Dependence 2011; 118: 470-4.

44.- ElSohly M: Chemical constituents of cannabis. En: Cannabis and Canabinoids: Pharmacology, Toxicology and Therapeutic Potential. Franjo Grotenhermen and Ethan B Russo, Editores. Harward Press, Inc, Alice Street, Binghamton NY 2013: Capítulo 3 p: 65-86.

45.- Fong T, Heymsfield S: Canabinoid-1 receptor inverse agonist: current understandisng of mechanism of acction and unasnswered questions. Int J Obes 2009; 33: 947$55 \mathrm{a}$. 